\title{
Simultaneous NIR/sub-mm observation of flare emission from Sagittarius $A^{*}$
}

\author{
A. Eckart ${ }^{1,2}$, R. Schödel ${ }^{3}$, M. García-Marín ${ }^{1}$, G. Witzel ${ }^{1}$, A. Weiss ${ }^{2}$, F. K. Baganoff ${ }^{4}$, M. R. Morris ${ }^{5}$, T. Bertram ${ }^{1}$, \\ M. Dovčiak ${ }^{6}$, W. J. Duschl ${ }^{7,8}$, V. Karas ${ }^{6}$, S. König ${ }^{1}$, T. P. Krichbaum ${ }^{2}$, M. Krips ${ }^{9,14}$, D. Kunneriath ${ }^{1,2}$, R.-S. Lu ${ }^{2,1}$, \\ S. Markoff ${ }^{10}$, J. Mauerhan ${ }^{5}$, L. Meyer ${ }^{5}$, J. Moultaka ${ }^{11}$, K. Mužić ${ }^{1}$, F. Najarro ${ }^{12}$, J.-U. Pott ${ }^{5,13}$, K. F. Schuster ${ }^{14}$, \\ L. O. Sjouwerman ${ }^{15}$, C. Straubmeier ${ }^{1}$, C. Thum ${ }^{14}$, S. N. Vogel ${ }^{16}$, H. Wiesemeyer ${ }^{17}$, \\ M. Zamaninasab ${ }^{1,2}$, and J. A. Zensus ${ }^{2}$
}

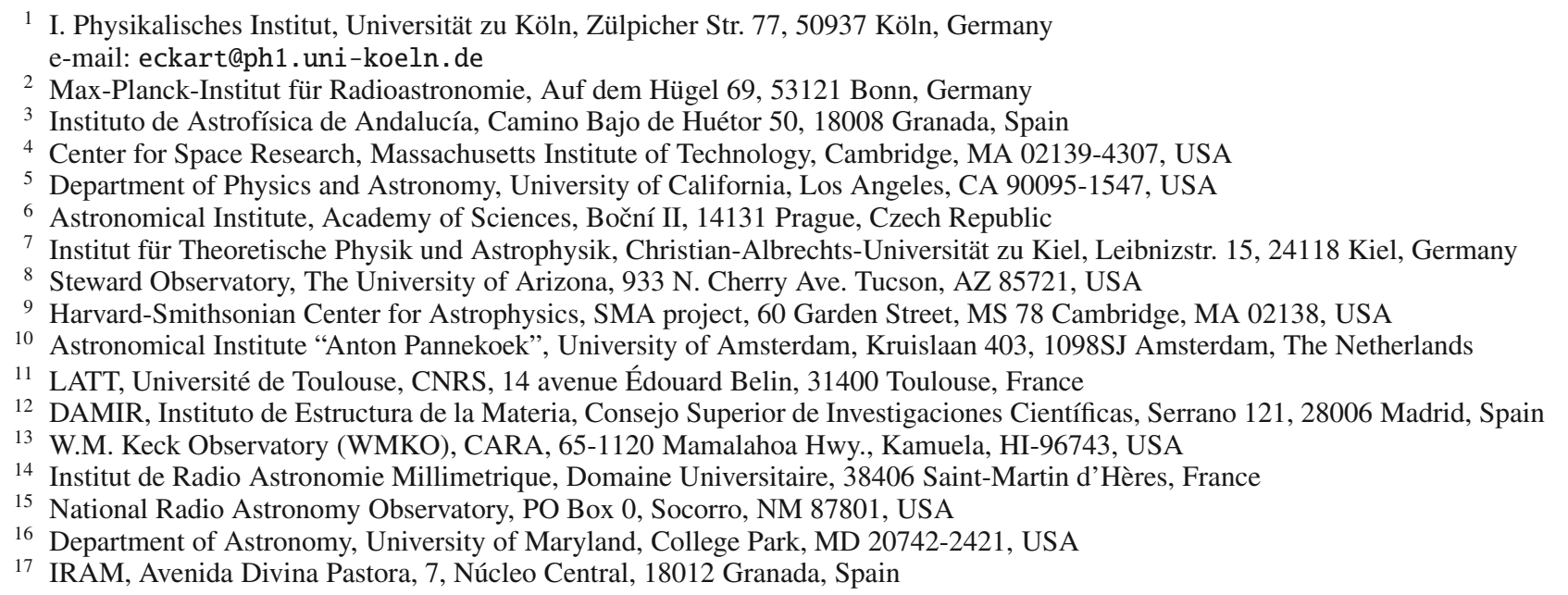

Received 6 September 2008 / Accepted 23 October 2008

\section{ABSTRACT}

\begin{abstract}
Context. We report on a successful, simultaneous observation and modeling of the sub-millimeter to near-infrared flare emission of the Sgr A* counterpart associated with the super-massive $\left(4 \times 10^{6} M_{\odot}\right)$ black hole at the Galactic center.

Aims. We study and model the physical processes giving rise to the variable emission of Sgr $\mathrm{A}^{*}$.

Methods. Our non-relativistic modeling is based on simultaneous observations that have been carried out on $03 \mathrm{June}, 2008$. We used the NACO adaptive optics (AO) instrument at the European Southern Observatory's Very Large Telescope and the LABOCA bolometer at the Atacama Pathfinder Experiment (APEX). We emphasize the importance of a multi-wavelength simultaneous fitting as a tool for imposing adequate constraints on the flare modeling.

Results. The observations reveal strong flare activity in the $0.87 \mathrm{~mm}(345 \mathrm{GHz})$ sub-mm domain and in the $3.8 \mu / 2.2 \mu \mathrm{m} \mathrm{NIR}$. Inspection and modeling of the light curves show that the sub-mm follows the NIR emission with a delay of $1.5 \pm 0.5 \mathrm{~h}$. We explain the flare emission delay by an adiabatic expansion of the source components. The derived physical quantities that describe the flare emission give a source component expansion speed of $v_{\exp } \sim 0.005 c$, source sizes around one Schwarzschild radius with flux densities of a few Janskys, and spectral indices of $\alpha=0.8$ to 1.8, corresponding to particle spectral indices $\sim 2.6$ to 4.6. At the start of the flare the spectra of these components peak at frequencies of a few THz.

Conclusions. These parameters suggest that the adiabatically expanding source components either have a bulk motion greater than $v_{\mathrm{exp}}$ or the expanding material contributes to a corona or disk, confined to the immediate surroundings of Sgr A*.
\end{abstract}

Key words. black hole physics - infrared: general - accretion, accretion disks - Galaxy: center - Galaxy: nucleus

\section{Introduction}

At the center of the Milky Way, at a distance of only about $8 \mathrm{kpc}$, stellar orbits have convincingly proven the existence of a super-massive black hole $(\mathrm{SMBH})$ of mass $\sim 4 \times 10^{6} M_{\odot}$ at the position of the compact radio, infrared, and X-ray source Sagittarius A* (Sgr A*; Eckart \& Genzel 1996, 1997; Eckart et al. 2002; Schödel et al. 2002; Eisenhauer et al. 2003; Ghez et al. 2000, 2005a). Additional strong evidence of an SMBH at the position of $\mathrm{Sgr} \mathrm{A}^{*}$ comes from the observation of rapid flare activity both in the X-ray and NIR wavelength domains (Baganoff et al. 2001; Genzel et al. 2003; Ghez et al. 2004; Eckart et al. 2006a-c). Due to its proximity, Sgr A* provides us with a unique opportunity to understand the physics and possibly the evolution of SMBHs at the nuclei of galaxies. Variability at radio through sub-millimeter wavelengths has been studied extensively, showing that variations occur on timescales from hours to years (e.g. Mauerhan et al. 2005; Eckart et al. 2006a; 
Table 1. Observation log.

\begin{tabular}{|c|c|c|c|c|}
\hline $\begin{array}{c}\text { Telescope } \\
\text { observing ID }\end{array}$ & Instrument & $\bar{\lambda}$ & $\begin{array}{l}\text { UT and JD } \\
\text { start time }\end{array}$ & $\begin{array}{l}\text { UT and JD } \\
\text { stop time }\end{array}$ \\
\hline VLT UT 4 & $\overline{\mathrm{NACO}}$ & $2.2 \mu \mathrm{m}$ & $\begin{array}{l}20083 \text { June } 08: 37: 23 \\
\text { JD } 2454620.8593\end{array}$ & $\begin{array}{l}\text { 3 June 09:58:59 } \\
\text { JD } 2454620.9159\end{array}$ \\
\hline VLT UT 4 & NACO & $3.8 \mu \mathrm{m}$ & $\begin{array}{l}20083 \text { June } 04: 41: 34 \\
\text { JD } 2454620.6955\end{array}$ & $\begin{array}{l}3 \text { June } 8: 20: 14 \\
\text { JD } 2454620.8474\end{array}$ \\
\hline APEX & LABOCA & $870 \mu \mathrm{m}$ & $\begin{array}{l}20083 \text { June 04:56:55 } \\
\text { JD } 2454620.7062\end{array}$ & $\begin{array}{l}3 \text { June } 10: 22: 55 \\
\text { JD } 2454620.9326\end{array}$ \\
\hline
\end{tabular}

Yusef-Zadeh et al. 2008; Marrone et al. 2008). Several flares have provided evidence of decaying millimeter and submillimeter emission following simultaneous NIR/X-ray flares. Although much effort has been invested in monitoring of the galactic center variability at these wavelengths, only a handful of simultaneous observations aimed at analyzing their correlation have been carried out until now (see for instance Eckart et al. 2006a, 2008a,b; Yusef-Zadeh et al. 2006a,b, 2007, 2008; Marrone et al. 2008).

Here we report on the first measurements of $\operatorname{Sgr} \mathrm{A}^{*}$ that successfully detected simultaneous flare emission in the nearinfrared and sub-millimeter domain using the ESO VLT and the APEX sub-mm telescopes ${ }^{1}$. In the second section we describe the observations and data reduction followed by an outline of the flare modeling in Sect. 3. We conclude with a discussion and summary in Sects. 4 and 5.

For optically thin synchrotron emission we refer throughout this paper to photon spectral indices $(\alpha)$ using the convention $S_{v} \propto v^{-\alpha}$ and to spectral indices $(p)$ of electron power-law distributions using $N(E) \propto E^{-p}$ with $p=(1+2 \alpha)$. The assumed distance to Sgr A* is $8 \mathrm{kpc}$ (Reid 1993), consistent with recent results (e.g. Lu et al. 2008a; Ghez et al. 2005a, 2008; Eisenhauer et al. 2003).

\section{Observations and data reduction}

The sub-mm regime is of special interest for simultaneous flare measurements, as it provides important constraints on the modeling. In this general wavelength range synchrotron source components that also radiate in the infrared domain become optically thick, and represent the dominant reservoir of photons that are then scattered to the X-ray domain through the inverse Compton process. On 3 June 2008, substantial progress was made in this matter: as part of a larger campaign, $\mathrm{Sgr} \mathrm{A}^{*}$ was simultaneously observed in the sub-millimeter and NIR wavelength domains using the European Southern Observatory facilities. These observations result in the first simultaneous successful detection of strongly variable emission in both wavelength domains. In the following subsections we describe the data acquisition and reduction for the individual telescopes. The basic details of the individual observing sessions are given in Table 1.

\subsection{The NIR data}

Near-infrared (NIR) observations of the Galactic center (GC) were carried out with the NIR camera CONICA and the adaptive

\footnotetext{
${ }^{1}$ Based on observations with the ESO telescopes at the Paranal Observatory under programs IDs:077.B-0028, 79.B-0084, and 81.B0648. The sub-mm data are based on observations with the Atacama Pathfinder Experiment (APEX). APEX is a collaboration between the Max-Planck-Institut für Radioastronomie, the European Southern Observatory, and the Onsala Space Observatory.
}

Table 2. Details of NIR observations.

\begin{tabular}{cccccc}
\hline \hline$\lambda$ & DIT & NDIT & $N$ & Pixel scale & Seeing \\
\hline $2.2 \mu \mathrm{m}$ & $10 \mathrm{~s}$ & 4 & 80 & 0.027 & $\sim 1^{\prime \prime} 0-1^{\prime \prime \prime} 4$ \\
$3.8 \mu \mathrm{m}$ & $0.2 \mathrm{~s}$ & 150 & 150 & $0{ }^{\prime} 027$ & $\sim 1^{\prime \prime} 1-11^{\prime \prime} 7$ \\
\hline
\end{tabular}

optics (AO) module NAOS (briefly "NACO") at the ESO VLT unit telescope 4 (YEPUN) on Paranal, Chile, during the night between 2 June and 3 June 2008. In all observations, the infrared wavefront sensor of NAOS was used to lock the AO loop on the NIR bright ( $K$-band magnitude $\sim 6.5$ ) supergiant IRS 7 , located about 5.6" north of Sgr A*. Details on integration times and approximate seeing during the observations are listed in Table 2. Here $\lambda$ is the central wavelength of the broad-band filter used. DIT is the detector integration time in seconds. NDIT is the number of exposures of integration time DIT that were averaged online by the instrument. $N$ is the number of images taken. The total integration time amounts to DIT $\times$ NDIT $\times N$. Seeing is the value measured by the Differential Image Motion Monitor (DIMM) on Paranal at visible wavelengths. It provides a rough estimate of atmospheric conditions during the observations. The atmospheric conditions (and consequently the AO correction) were stable during the observations.

All observations in the $L^{\prime}$-band $(3.8 \mu \mathrm{m})$ and $K$-band $(2.2 \mu \mathrm{m})$ were dithered to cover a larger area of the GC by mosaic imaging. The sky background for the $K$-band observations was extracted from the median of stacks of dithered exposures of a dark cloud - a region practically empty of stars - a few arcminutes to the northwest of $\mathrm{Sgr} \mathrm{A}^{*}$. The $L^{\prime}$-band observations were interspersed with frequent off-target exposures that served to determine the rapidly varying background in this filter. All exposures were sky subtracted, flat-fielded, and corrected for dead or bad pixels. Subsequently, PSFs were extracted from these images with StarFinder (Diolaiti et al. 2000). The images were deconvolved with the Lucy-Richardson (LR) algorithm. Beam restoration was carried out with a Gaussian beam of FWHM corresponding to the respective wavelength. The final resolution at 2.2 and $3.8 \mu \mathrm{m}$ is about 60 and 104 milli-arcsec (mas), respectively.

The flux densities of the sources were measured by aperture photometry with circular apertures of 52 mas radius and corrected for extinction, using $A_{K}=2.8$, and $A_{L^{\prime}}=1.8$. The relative flux density calibration was carried out using known $K$ and $L^{\prime}$-band flux densities of IRS16SW, IRS16C, IRS16NE, and IRS2 1 by Blum et al. (1996). This results in $K$ - and $L^{\prime}$-band flux densities of the high velocity star S2 of $22 \pm 1 \mathrm{mJy}$ and $9 \pm 1 \mathrm{mJy}$, respectively, which compare well with magnitudes and fluxes for S2 quoted by Ghez et al. (2005b) and Genzel et al. (2003). The relative photometry for Sgr A* was done with the known fluxes and positions of 9 sources within 1".6 of Sgr A*. The measurement uncertainties for Sgr A* were obtained on the 


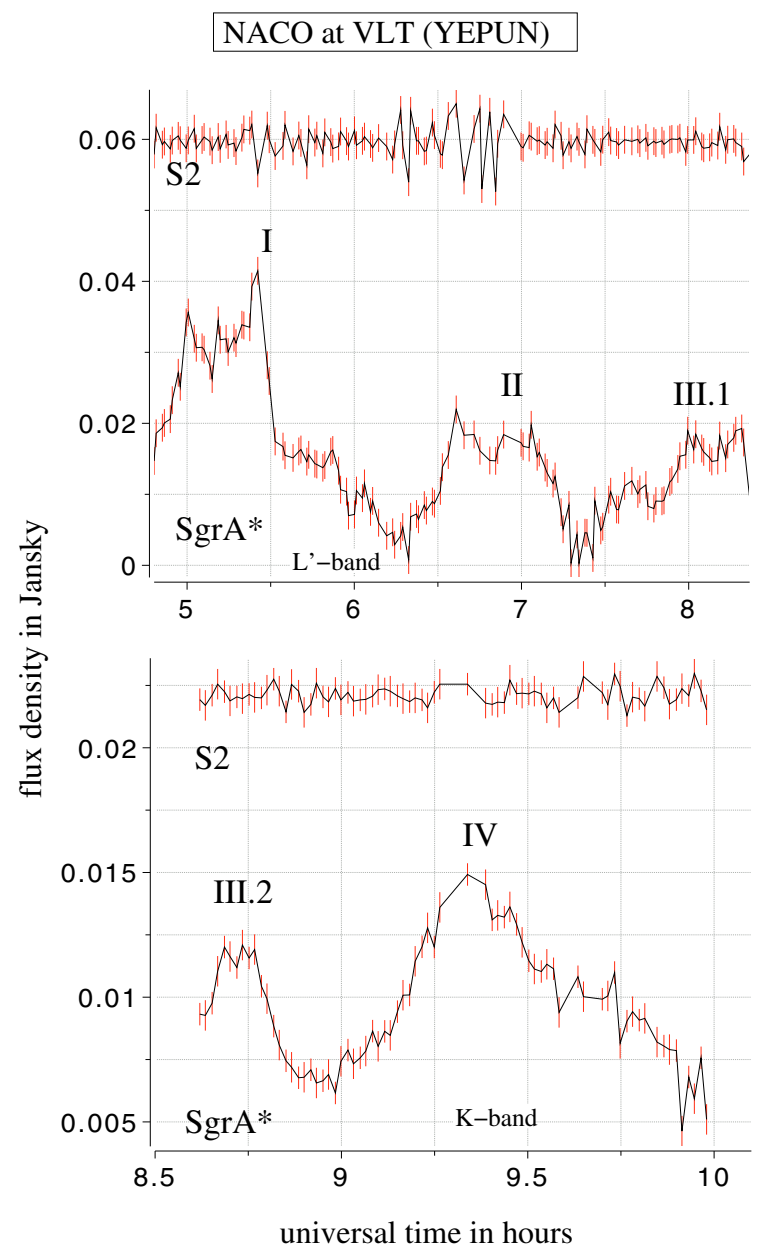

Fig. 1. The infrared light curve of Sagittarius A* on 3 June 2008. The data are represented by vertical red bars $( \pm 1 \sigma)$ and a black connection line between them. Upper panel: $L^{\prime}$-band flare events. For a better representation we added a constant value of $50 \mathrm{mJy}$ to the light curve for the reference star. Lower panel: the $K$-band flare emission immediately following the $L^{\prime}$-band observations. In this case no offset was added to the light curve for the reference star.

reference star S2. The background flux in the immediate vicinity of Sgr A* was obtained by averaging the measurements at six random locations in a field located about $0^{\prime} .6$ west of Sgr A* that is free of obvious stellar sources.

The resulting light curves of Sgr A* and S2 (as a constant reference star) are shown in Fig. 1.

\subsection{The sub-mm data}

The Galactic center $870 \mu \mathrm{m}$ data were taken with the newly commissioned LABOCA bolometer array, located on the Atacama Pathfinder EXperiment (APEX) telescope at the Llano Chajnantor, Chile, at an altitude of $5105 \mathrm{~m}$. The observations were carried out as part of global multi-wavelength monitoring program in May/June 2008. The radiation is collected by the APEX telescope and fed through conical horns to the detectors of the Large APEX Bolometer Camera (LABOCA), an array of 295 composite bolometers that are extremely sensitive to continuum radiation. With a total bandwidth of about $60 \mathrm{GHz}$ the system is optimized for the $345 \mathrm{GHz}$ atmospheric window.

The full width half maximum (FWHM) of the point spread function (PSF) at $870 \mu \mathrm{m}$ is $\sim 19^{\prime \prime}$. To allow for the best possible reconstruction of the complex sub-mm emission in the GC region we used on the fly (OTF) maps perpendicular to the galactic plane (Weiss et al. 2008; Siringo et al. 2007). The maps were observed with a scanning speed of $3^{\prime} /$ s modulating the source signals even for extended structures into the useful post-detection frequency band of LABOCA $(0.1-12.5 \mathrm{~Hz})$. To avoid scanning artefacts in the reconstruction of the extended thermal emission surrounding Sgr A*, we derived and averaged maps from the data with an inclination of $-10^{\circ}, 0^{\circ}$ and $+10^{\circ}$ with respect to an axis orthogonal to the galactic plane. The mapping steps perpendicular to the scanning direction was $30^{\prime \prime}$ and the integration time $280 \mathrm{~s}$, yielding an rms noise level of $\sim 150 \mathrm{mJy} / \mathrm{beam}$ for each map.

Immediately after each galactic center map, either G10.62 or IRAS16293-2422 were observed as secondary calibrator sources. We reduced the data with the $\mathrm{BoA}^{2}$ software package. The data reduction process included correction for atmospheric zenith opacity (towards the Galactic center $\tau \sim 0.3$ up to 0.7 ), flat-fielding, de-spiking, correlated sky noise removal, and the removal of further correlated noise due to instrumental effects.

After correcting for pointing offsets between individual maps (determined from the position of Sgr $\mathrm{A}^{*}$ in each map) all scans were co-added to obtain high $\mathrm{S} / \mathrm{N}, 48^{\prime} \times 25^{\prime}$ fully sampled maps of the sub-mm emission in the GC regions. From this combined map the point source Sgr A* was modeled with a Gaussian and taken out. The uncertainty in that process will result in a small offset of the sub-mm light curve. We estimate that this effect is less than $10 \%$ of the peak flux density of SgrA*. In this way we created a model image of the extended $870 \mu \mathrm{m}$ emission surrounding Sgr A* with Sgr A* effectively set in an off state (see Fig. 2).

Each data point of the Sgr A* sub-mm light curve (see Fig. 3) was derived from the model subtracted maps, modeling a Gaussian source and deriving the peak. In order to provide a stable and consistent calibration we used the $\tau$ values derived from the sky dip and radiometer measurements, as well as secondary flux calibrators and a comparison to the reference map. Between the individual observing epochs the flux densities of the secondary flux calibrators vary by $\leq 10 \%$. From a comparison of different reference sources, we estimate the relative pointto-point uncertainty in the calibration of the light curve measurements to be of the order of $4 \%(1 \sigma)$. The remaining flux density variations in the light curve in Fig. 3 may arise from residual fast and uncompensated opacity or pointing changes.

\section{Flare analysis}

\subsection{Adiabatically expanding source components}

Our basic assumption to model the sub-mm light curves is the presence of an expanding uniform blob of relativistic electrons with an energy spectrum $n(E) \propto E^{-p}$ threaded by a magnetic field. As a consequence of the blob expansion, the magnetic field inside the blob declines as $R^{-2}$, the energy of relativistic particles as $R^{-1}$ and the density of particles as $R^{-3}$ (van der Laan 1966). The synchrotron optical depth at frequency $v$ then scales as

$\tau_{v}=\tau_{0}\left(\frac{v}{v_{0}}\right)^{-(p+4) / 2}\left(\frac{R}{R_{0}}\right)^{-(2 p+3)}$

and the flux density scales as

$S_{v}=S_{0}\left(\frac{v}{v_{0}}\right)^{5 / 2}\left(\frac{R}{R_{0}}\right)^{3} \frac{1-\exp \left(-\tau_{v}\right)}{1-\exp \left(-\tau_{0}\right)}$.

${ }^{2}$ BoA: http://www.astro.uni-bonn.de/boawiki/Boa 

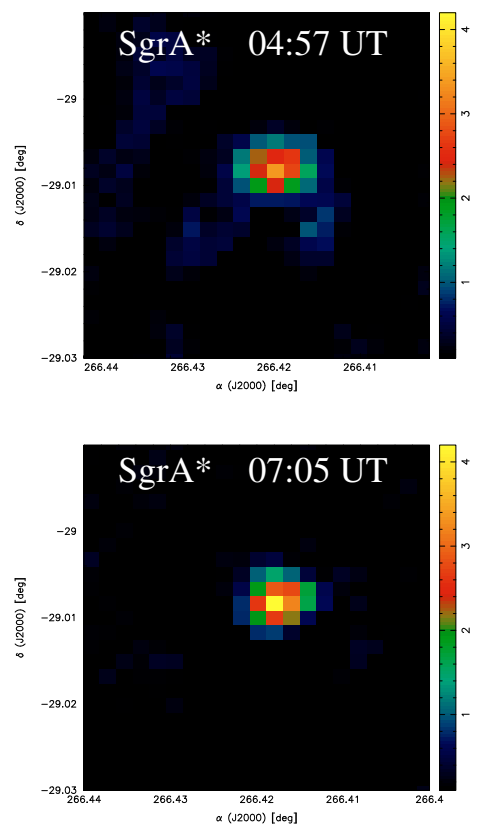

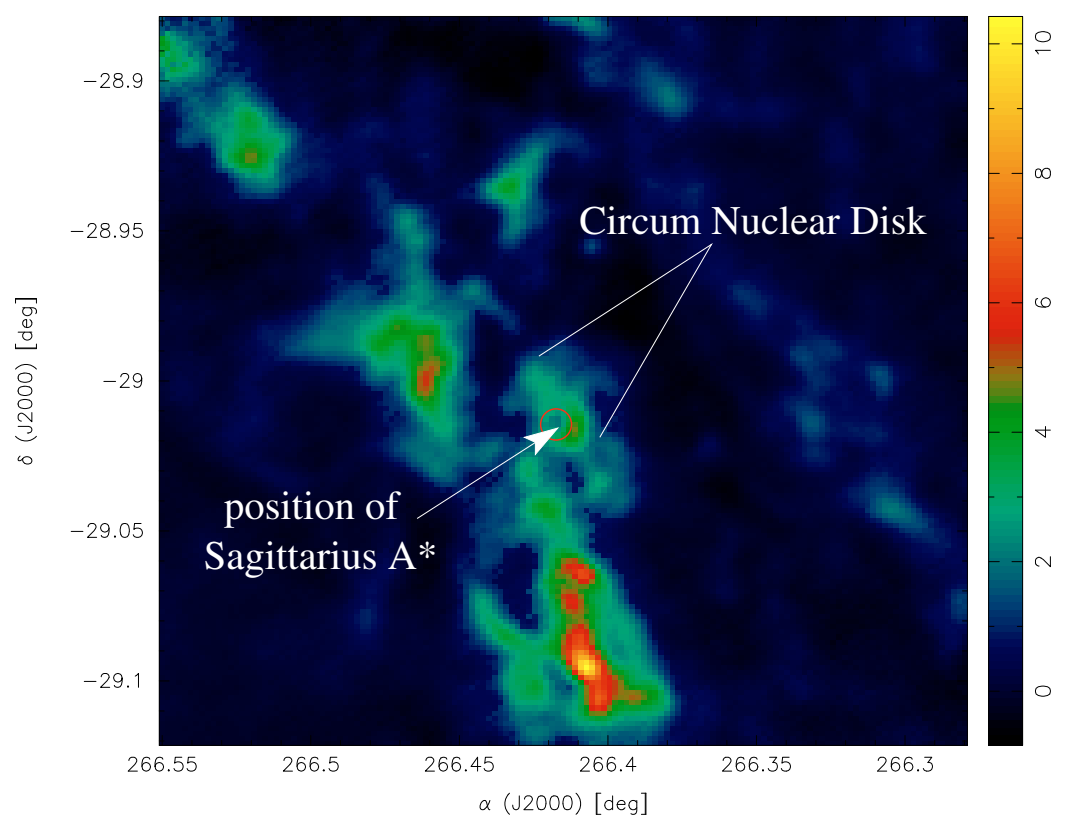

Fig. 2. Left: Sagittarius $\mathrm{A}^{*}$ at the beginning and the peak of the APEX measurements. Right: a section of the larger map of the Galactic center. The location of Sagittarius A* is indicated by a circle. Sagittarius A* has been subtracted out as this map is used as a reference for the extended structure. The colors code the flux density in Janskys.

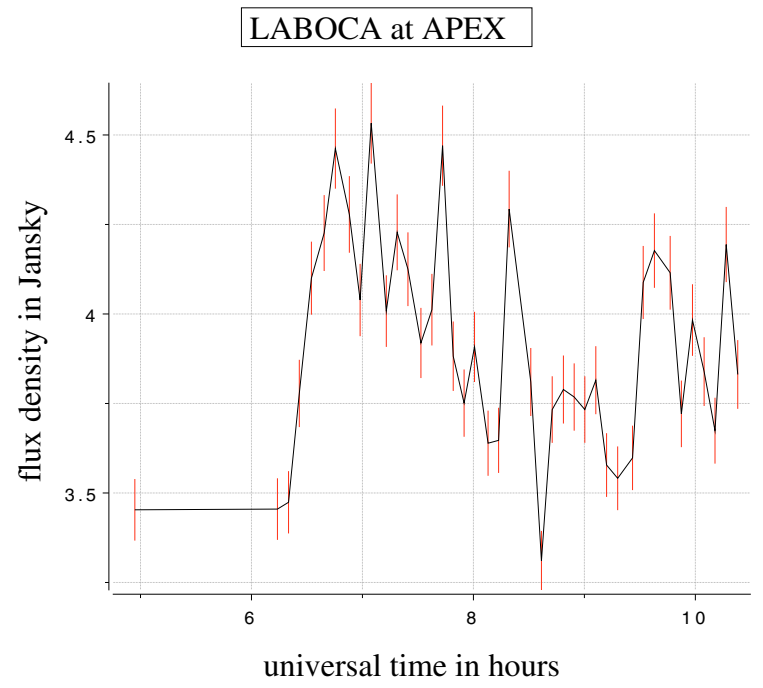

Fig. 3. The sub-mm light curve of Sagittarius A* on 3 June 2008. The data are represented by vertical red bars $( \pm 1 \sigma$ in length) and a black connection line between them.

Here $R_{0}, S_{0}$, and $\tau_{0}$ are the size, flux density, and optical depth at the peak frequency of the synchrotron spectrum $v_{0}$. The goal of the present model is to combine the description of an adiabatically expanding cloud with a synchrotron self-Compton formalism, as this is the most likely physical scenario to explain the delay between the sub-mm and the simultaneous near-IR and $\mathrm{X}$-ray peaks. Thus, we use the definition of $\tau_{0}$ as the optical depth corresponding to the frequency at which the flux density is a maximum (van der Laan 1966), rather than the definition of $\tau_{0}$ as the optical depth at which the flux density for any particular frequency peaks (Yusef-Zadeh et al. 2006b). This implies that $\tau_{0}$ depends only on $p$ through the condition

$\mathrm{e}^{\tau_{0}}-\tau_{0}(p+4) / 5-1=0$ and ranges from, e.g., 0 to 0.65 as $p$ ranges from 1 to 3 . Therefore, given the particle energy spectral index $p$ and the peak flux $S_{0}$ in the light curve at some frequency $v_{0}$, this model predicts the variation in flux density at any other frequency as a function of the expansion factor $\left(R / R_{0}\right)$.

Finally, a model for $R(t)$ is required to convert the dependence on radius to time: we adopt a simple linear expansion at constant expansion speed $v_{\text {exp }}$, so that $R-R_{0}=v_{\exp }\left(t-t_{0}\right)$. For $t \leq t_{0}$ we have made the assumption that the source has an optical depth that equals its frequency dependent initial value $\tau_{v}$ at $R=R_{0}$. So in the optically thin part of the source spectrum the flux initially increases with the source size at a constant $\tau_{v}$ and then decreases due to the decreasing optical depth as a consequence of the expansion. For the $\sim 4 \times 10^{6} M_{\odot}$ super-massive black hole at the position of Sgr $\mathrm{A}^{*}$, one Schwarzschild radius is $R_{\mathrm{S}}=2 \mathrm{GM} / \mathrm{c}^{2} \sim 10^{10} \mathrm{~m}$ and the velocity of light corresponds to about $100 R_{\mathrm{s}}$ per hour. For $t>t_{0}$ the decaying flank of the curve can be shifted towards later times by first, increasing the turnover frequency $v_{0}$ or the initial source size $R_{0}$, and second, by lowering the spectral index $\alpha_{\text {synch }}$ or the peak flux density $S_{0}$. Increasing the adiabatic expansion velocity $v_{\exp }$ shifts the peak of the light curve to earlier times. Adiabatic expansion will also result in a slower decay rate and a longer flare timescale at lower frequencies.

\subsection{Modeling the flare emission}

The combined $L^{\prime}$ - and $K$-band data show violently variable emission with at least 4 prominent flare events (I-IV, see Fig. 1). As we switched from $L$ - to $K$-band during an ongoing flare we attribute the $L$-band flare Sect. 3.1 and the $K$-band flare Sect. 3.2 to the same NIR flare III with a duration from about 7:30 UT to about 9:00 UT.

As can be seen from the first data points in Fig. 3, the sub-mm data start with a low flux density at the time of the first NIR flare (Fig. 1). The gap shown in the sub-mm data between 
Table 3. Here we list data of the flares observed by the VLT and APEX on 3 June 2008. We list the estimated start and stop times, the full width at zero power (FWZP) and full width at half maximum $(F H W M)$ values, as well as estimates of the flare peaks and light curve minima which may be considered as offsets. The flare peak flux densities are given in $10^{-3}$ Jansky. Flux density uncertainties are given as their $1 \sigma$ values.

\begin{tabular}{llcccccccc}
\hline \hline $\begin{array}{l}\text { Flare } \\
\text { I.D. }\end{array}$ & $\begin{array}{l}\text { Spectral } \\
\text { domain }\end{array}$ & $\begin{array}{c}\text { Flare start } \\
\text { time }\end{array}$ & $\begin{array}{c}\text { Flare stop } \\
\text { time }\end{array}$ & $\begin{array}{c}\text { Flare peak } \\
\text { time }\end{array}$ & $\begin{array}{c}\text { FWZP } \\
(\mathrm{min})\end{array}$ & $\begin{array}{c}F W H M \\
(\mathrm{~min})\end{array}$ & $\begin{array}{c}\text { Total } \\
\text { peak } \\
(\mathrm{mJy})\end{array}$ & $\begin{array}{c}\text { Flare } \\
\text { peak } \\
(\mathrm{mJy})\end{array}$ & $\begin{array}{c}\text { Offset } \\
(\mathrm{mJy})\end{array}$ \\
\hline I & NIR $L^{\prime}$-band & $<04: 43$ & $06: 20 \pm 10 \mathrm{~min}$ & $05: 15 \pm 5 \mathrm{~min}$ & $>90$ & $40 \pm 5$ & $32 \pm 2$ & $\geq 29$ & $\leq 3$ \\
II & NIR $L^{\prime}$-band & $06: 20 \pm 10 \mathrm{~min}$ & $07: 20 \pm 10 \mathrm{~min}$ & $06: 50 \pm 5 \mathrm{~min}$ & $60 \pm 10$ & $40 \pm 5$ & $17 \pm 2$ & $\geq 14$ & $\leq 3$ \\
III 1 & NIR $L^{\prime}$-band & $07: 20 \pm 10 \mathrm{~min}$ & $>8: 20$ & $08: 10 \pm 5 \mathrm{~min}$ & $>55$ & $>25$ & $17 \pm 2$ & $\geq 14$ & $\leq 3$ \\
III 2 & NIR $K$ s-band & $<8: 20$ & $08: 54 \pm 10 \mathrm{~min}$ & $08: 43 \pm 5 \mathrm{~min}$ & $>20$ & $10 \pm 5$ & $12 \pm 1$ & $\geq 6$ & $\leq 6$ \\
IV & NIR $K$ s-band & $08: 54 \pm 10 \mathrm{~min}$ & $09: 48 \pm 10 \mathrm{~min}$ & $09: 20 \pm 5 \mathrm{~min}$ & $55 \pm 10$ & $22 \pm 5$ & $14 \pm 1$ & $\geq 8$ & $\leq 6$ \\
- & sub-mm & $\leq 06: 00$ & $08: 50 \pm 20 \mathrm{~min}$ & $07: 00 \pm 15 \mathrm{~min}$ & $>220$ & $>140$ & $4250 \pm 200$ & $850 \pm 200$ & $\sim 3400$ \\
\hline
\end{tabular}

$5 \mathrm{~h}$ and $6 \mathrm{~h}$ occurred during culmination of $\operatorname{Sgr} \mathrm{A}^{*}$, when the source rises above the elevation limit $\left(80^{\circ}\right)$ for observations with APEX. In the sub-mm domain the variable emission is dominated by a single flare that is wider than the individual NIR flares (see Table 3). The so far six reported coordinated SgrA* measurements that include sub-mm data (Eckart et al. 2006b; Yusef-Zadeh et al. 2006b; Marrone et al. 2008, including this work) have shown that the observed submillimeter flares follow the largest event observed at the shorter wavelengths (NIR/X-ray; see detailed discussion in Marrone et al. 2008). We therefore assume that the sub-millimeter flare presented here is related to the observed IR flare events.

Separating the variable sub-mm emission: in order to separate the rapid intra-day flare emission from the sub-mm emission that varies much slower on time scales of days, a constant flux density level has been subtracted. In most instances Sgr A* has been tracked over long time spans on each day to obtain light curves. We have determined the median and median deviation of the lowest flux density measurements of the obtained light curves. This allows us to determine a flux density contribution that is constant or only slowly variable on time scales much longer than a single day. For this purpose we compared 5 APEX light curves taken with LABOCA between May 27 and June 3 (centered at a wavelength of $0.87 \mathrm{~mm}$, García-Marín 2008, in prep.) as well as $6 \mathrm{CSO}$ measurements at $0.85 \mathrm{~mm}$ wavelength by Yusef-Zadeh et al. (2006a, 2008) and 2 SMA light curves at $1.3 \mathrm{~mm}$ wavelength by Marrone et al. (2008). The SMA interferometer resolves out extended emission on scales of a few arcesonds. In the case of the APEX measurements a model of the extended emission on scales of $19^{\prime \prime}$ is subtracted. The flux density offsets of the different light curves are very similar. Therefore we attribute them to be intrinsic to the emission of $\mathrm{Sgr} \mathrm{A}^{*}$. The comparison results in a constant offset value of $3.0 \pm 0.5 \mathrm{Jy}$. For the the lowest submillimeter flux densities obtained on June 3 we find a constant contribution of $3.4 \mathrm{Jy}$. This value is in very good agreement with the median derived above. Therefore we subtracted it from the light curve in order to extract the intra-day variable part of the sub-mm data.

Adiabatic expansion modeling: we model the 4 flare events I-IV and the smoothed version of the sub-millimeter data with 4 adiabatically expanding source components (see dashed line in Fig. 4. The calculations show that the initial synchrotron self-absorption cutoff frequency of the source components is a critical parameter that allows us to distinguish between separate models. From our modeling we can constrain the cutoff frequency between 1 and $2.5 \mathrm{THz}$ (model A:2.5 THz, model B:2 THz and model $\mathrm{C}: 1 \mathrm{THz}$ ), and establish from

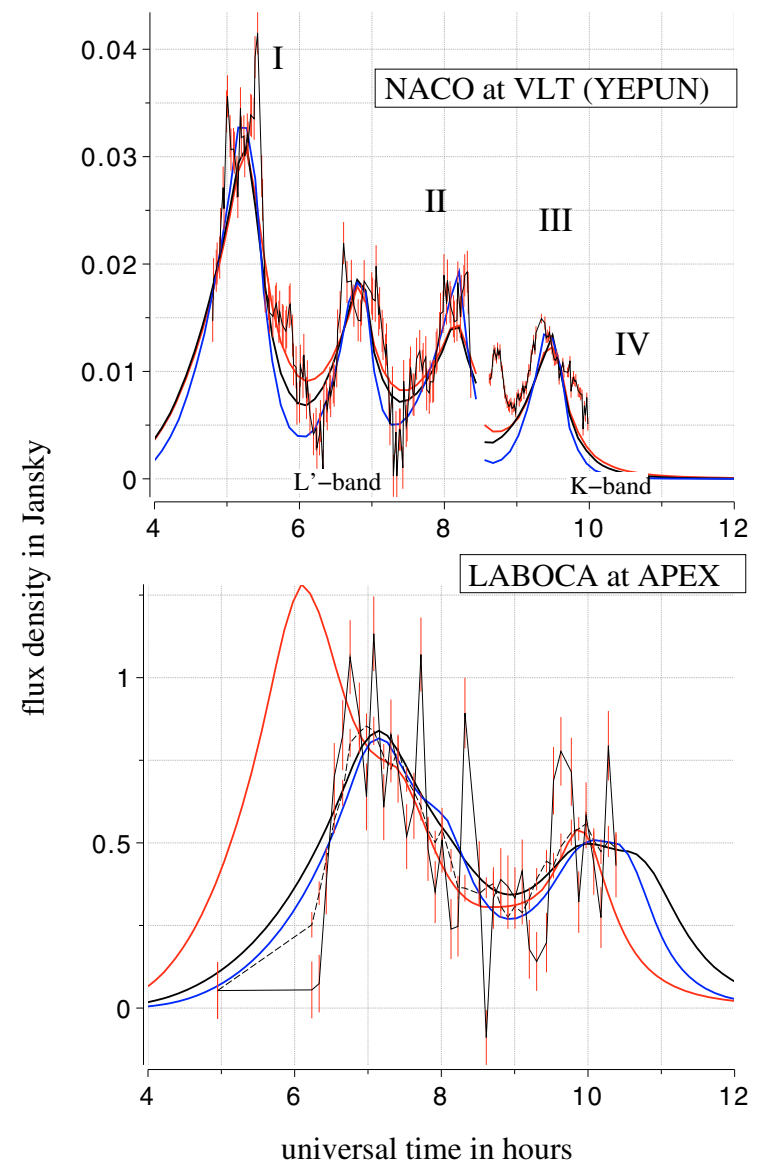

Fig. 4. The infrared (top) and sub-mm (bottom) light curve of Sagittarius $\mathrm{A}^{*}$. The data are represented by vertical red bars $( \pm 1 \sigma)$ and a black connection line between them. The dashed line represents a smoothed version of the data (after application of a 7 point sliding average for all data points exept the first). The models as described in the text and in Table 4 (blue, black, red for models A, B, C, respectively) are shown as solid lines (blue: $\sim 2.5 \mathrm{THz}$; black: $\sim 2 \mathrm{THz}$; red: $\sim 1 \mathrm{THz}$ cutoff frequency in the source component spectra).

inspection and modeling of the light curves a delay between the sub-mm and NIR emission of $1.5 \pm 0.5 \mathrm{~h}$. Model fits to the variable sub-mm emission are shown in comparison to the data in Fig. 4. The corresponding model parameters are given in Table 4. To select the intra-day variable part of the sub-mm light curve a flux density of $3.4 \mathrm{Jy}$ has been subtracted from the sub-mm data in Fig. 4. This amount is attributed to more extended (many Schwarzschild radii) source components. We attribute the time difference between the NIR and sub-mm flares 
Table 4. Source component parameters for the adiabatic expansion model of the 3 June, 2008 flare. The flare times $t_{0}$ are given with respect to the peak of the brighter NIR flares labeled I, II, III, IV. For the adiabatic expansion velocity $v_{\text {exp }}$, the optically thin spectral index $\alpha_{\text {synch }}$ and the cutoff frequency $v_{0}$ the uncertainties were derived over the entire data set. In addition to $v_{\exp }$ the $\mathrm{R}_{0}$ values are responsible for the position and width of the infrared flares peaks in time. Different values for $\alpha_{\text {synch }}$ are requred to match the infrared flux densities. The derivation of uncertainties and reduced $\chi^{2}$ values is described in the text. Model B is the preferred model (see text).

\begin{tabular}{|c|c|c|c|c|c|c|c|c|}
\hline $\begin{array}{l}\text { Model } \\
\text { label }\end{array}$ & $\begin{array}{l}\text { Flare } \\
\text { label }\end{array}$ & $\begin{array}{l}t_{0} \\
\text { hours }\end{array}$ & $\begin{array}{l}v_{\exp } \\
\text { in } c\end{array}$ & $\begin{array}{r}S_{\text {max,obs }} \\
\text { [Jy] }\end{array}$ & 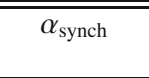 & $\overline{\overline{R_{0}}}$ & $\begin{array}{c}v_{0} \\
{[\mathrm{THz}]}\end{array}$ & $\begin{array}{l}\text { Reduced } \\
\chi^{2} \text { value }\end{array}$ \\
\hline \multirow[t]{4}{*}{$\overline{\mathrm{A}}$} & $\mathrm{I}$ & $5.3 \pm 0.2$ & $0.0040 \pm 0.0020$ & $11.4 \pm 3.6$ & $1.77 \pm 0.20$ & $1.1 \pm 0.10$ & $2.50 \pm 0.45$ & \multirow[t]{4}{*}{2.64} \\
\hline & II & $6.9 \pm 0.5$ & " & $4.8 \pm 3.5$ & $1.67 \pm 0.10$ & $0.7 \pm 0.40$ & " & \\
\hline & III & $8.2 \pm 0.4$ & " & $5.6 \pm 3.0$ & $1.77 \pm 0.10$ & $1.0 \pm 0.70$ & " & \\
\hline & IV & $9.5 \pm 0.2$ & $"$ & $4.1 \pm 2.0$ & $1.45 \pm 0.10$ & $0.6 \pm 0.50$ & $"$ & \\
\hline \multirow[t]{4}{*}{ B } & I & $5.3 \pm 0.2$ & \multirow{4}{*}{$0.0045 \pm 0.0020$} & $7.8 \pm 1.5$ & $1.57 \pm 0.10$ & $1.3 \pm 0.30$ & \multirow{4}{*}{$2.00 \pm 0.45$} & \multirow[t]{4}{*}{1.82} \\
\hline & II & $6.9 \pm 0.5$ & & $1.4 \pm 1.5$ & $1.25 \pm 0.10$ & $0.9 \pm 0.60$ & & \\
\hline & III & $8.2 \pm 0.4$ & & $3.3 \pm 1.6$ & $1.56 \pm 0.10$ & $1.2 \pm 0.70$ & & \\
\hline & IV & $9.4 \pm 0.2$ & & $2.8 \pm 0.5$ & $1.33 \pm 0.10$ & $0.9 \pm 0.50$ & & \\
\hline \multirow[t]{4}{*}{$\mathrm{C}$} & I & $5.3 \pm 0.2$ & \multirow{4}{*}{$\begin{array}{c}0.0053 \pm 0.0020 \\
"\end{array}$} & $4.0 \pm 1.3$ & $1.15 \pm 0.30$ & $1.3 \pm 0.60$ & \multirow{4}{*}{$\begin{array}{c}1.00 \pm 0.30 \\
" \\
" \\
"\end{array}$} & \multirow[t]{4}{*}{2.32} \\
\hline & II & $6.9 \pm 0.5$ & & $1.3 \pm 1.0$ & $1.05 \pm 0.20$ & $1.0 \pm 0.50$ & & \\
\hline & III & $8.2 \pm 0.5$ & & $0.5 \pm 1.3$ & $0.85 \pm 0.20$ & $1.2 \pm 0.30$ & & \\
\hline & IV & $9.4 \pm 0.2$ & & $1.4 \pm 0.3$ & $1.00 \pm 0.20$ & $0.7 \pm 0.40$ & & \\
\hline
\end{tabular}

to an adiabatic expansion of synchrotron source components with an expansion speed of about $0.5 \%$ of the speed of light $\left(1500 \mathrm{~km} \mathrm{~s}^{-1}\right)$. While the sub-mm light curve is modeled by the evolving optically thick part of the spectra, the NIR light curve is modeled through the optically thin part of the spectra. We find that the flares are associated with source components that have initial sizes of the order of one Schwarzschild radius and spectra that peak around $2 \mathrm{THz}$. The flux densities are a few Janskys and the spectral indices are optically thin between the sub-mm and the infrared with $0.6 \leq \alpha \leq 1.5$. Models with significantly different expansion speeds or source sizes fail to represent either the extent or shape of the observed flare features.

Models A and C represent border cases: for higher cutoff frequencies than the one used in model $\mathrm{A}$, the required flare fluxes at the $\mathrm{THz}$ turnover frequencies become increasingly higher to account for the sub-mm emission after adiabatic expansion. At the same time, the spectral index becomes increasingly steeper in order to meet the NIR flux densities. Towards cutoff frequencies well above $2.5 \mathrm{THz}$ the spectral index will become steeper than what has been measured in the NIR (Eisenhauer et al. 2005; Hornstein et al. 2006; Gillessen et al. 2006; Krabbe et al. 2006). On the other hand the mean spectral index for the component in model $\mathrm{C}$ is closer to the value of $0.6 \pm 0.2$ obtained by Hornstein et al. (2007), but the predicted source component labeled $I$ shows already an unacceptable discrepancy between the flux density observed with APEX at 04:56 UT. This discrepancy increases with decreasing cutoff frequency. Our favored model B is presented in Fig. 5. It has an intermediate cutoff frequency, shows an overlap in parameters with models $\mathrm{A}$ and $\mathrm{C}$, and consistently reproduces the flares in the sub-mm and NIR wavelength domains. With a deviation of about $3-4 \sigma$, the agreement of the spectral indicies with results by Hornstein et al. (2007) lies between that of model A and $\mathrm{C}$. The spectral indices are in good agreement with that obtained for $\alpha_{\mathrm{NIR} / \mathrm{X} \text {-ray }} \sim 1.3$ by Eckart et al. (2004, 2006a). This is consistent with the fact that the optically thin synchrotron spectral index (sub-mm to NIR) is expected to equal the broad band spectral index of the SSC spectrum.

SSC modeling as an additional constraint: we use a synchrotron model with an optically thin spectral index $\alpha$ and relativistic electrons with $\gamma_{\mathrm{e}} \sim 10^{3}$ following Gould (1979) and

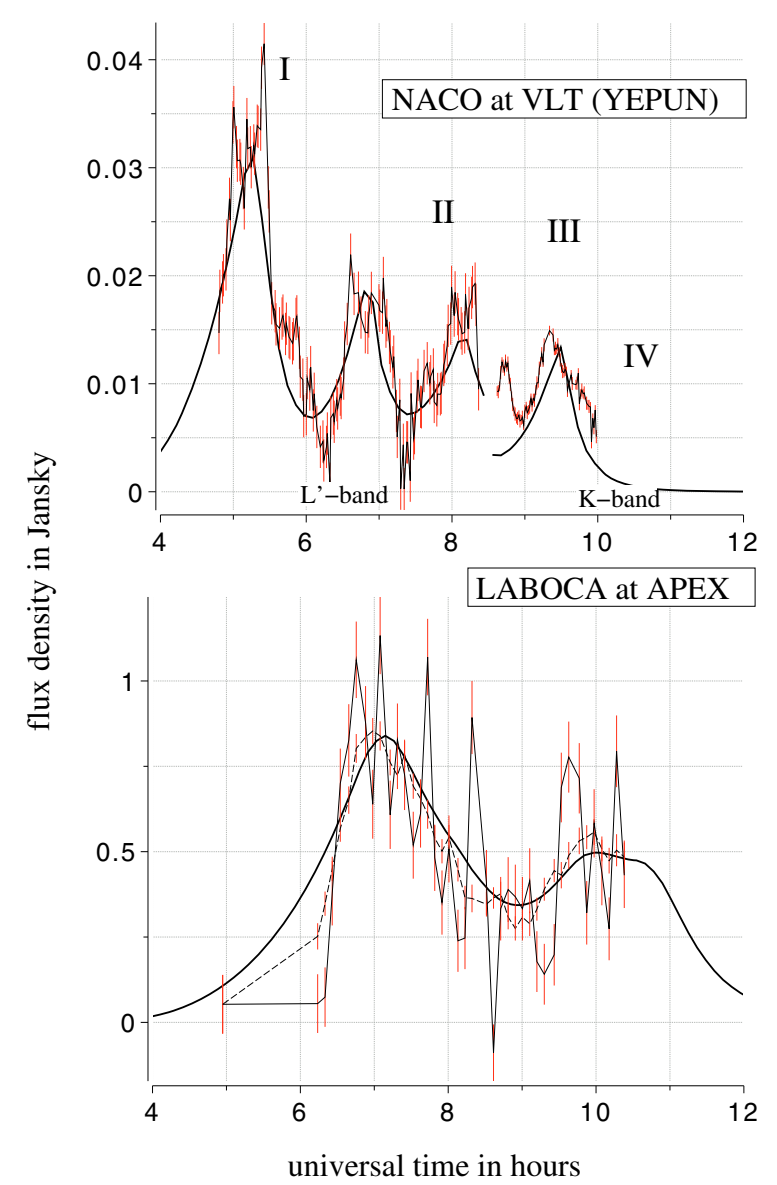

Fig. 5. Our favored model B compared to the infrared (top) and sub$\mathrm{mm}$ (bottom) light curve of Sagittarius A*. The data are represented by vertical red bars $( \pm 1 \sigma)$ and a black connection line between them. For furher explanation see caption of Fig. 4.

Marscher (1983). In the context of Sgr $A^{*}$ this model has been described by Eckart et al. (2004, 2008a). This formalism allows us to estimate the Synchrotron Self Compton (SSC) $\mathrm{X}$-ray flux densities and to compare them with values that have been measured before. In addition it allows us to estimate the 
SSC contribution of the NIR flux density and the magnetic field. In our adiabatic expansion modeling we have assumed that the NIR flux density is dominated by the synchrotron emission of the $\mathrm{THz}$ peak expanding synchrotron component. A strong excess in SSC NIR emission would violate that assumption and would require modeling the (unfortunately) unknown X-ray flux density as well. The magnetic field can be obtained via $B \sim \theta^{4} v_{\mathrm{m}}^{5} S_{\mathrm{m}}^{-2}$ as a direct result of the synchrotron source parameters. Previous models of the Sgr A* emission have resulted in magnetic field strengths of a few up to 70 Gauss (e.g. Eckart et al. 2006a, 2008a; Yusef-Zadeh et al. 2008; Marrone et al. 2008). The source components assumed for the adiabatic expansion model should not violate these results.

Our SSC calculations, that reveal typically observed X-ray flux densities in the range of a few 10 to $100 \mathrm{nJy}$ with magnetic fields of a few $10 \mathrm{G}$, indicate that the best solution lies between models B and C in Table 4. For model A we find that the SSC contribution of the NIR flux density becomes comparable to the synchrotron contribution. A correspondingly lower predicted NIR synchrotron contribution would require even steeper spectral indices. The SSC calculations, however, already indicate that the spectral indices and cutoff frequencies in model $\mathrm{A}$ need to be lower by $\Delta \alpha \sim 0.2$ and $\Delta v$ of a few $100 \mathrm{GHz}$ in order to give reasonable solutions that agree with typically observed and derived X-ray flux densities and magnetic fields. Therefore model A clearly appears less preferable.

Quality of the fit: sub-structures deviate by up to 4 times the measurement uncertainties from the overall structure described by flares I to IV. In order to estimate the fit quality we scale the assumed uncertainties to within which we can model the NIR data to $4 \sigma$. To compare the NIR and APEX data with the adiabatic expansion models, we calculated reduced $\chi^{2}$ values. Models with a large number of flare or source components would increase the number of free parameters and allow us to obtain better reduced $\chi^{2}$ values for the fits However, more complex models are currently difficult to justify. Following the ratio of the 41 APEX to 208 NIR data points, we increased the weight of the APEX data by a factor of 4 . With a total of 4 components (each with a time $t_{0}$, peak flux $S_{0}$, size $R_{0}$, and spectral index), a common cutoff frequency, sub-mm flux offset and expansion velocity $v_{\exp }$ we obtain a total of 19 free parameters. As shown in the Figs. 1 to 5 the $L$ - and $K$-band data were compared to the $L$ - and $K$-band model predictions, respectively. For our favorite model we calculated the uncertainties of the model parameters by varying them until the reduced $\chi$-values increase by $\Delta \chi \sim 0.5$. This corresponds to an increase across either of the 4 individual flare intervals in the sub-mm or the NIR domain of $\Delta \chi \sim 1.0$, corresponding to a confidence level of about $68.5 \%$ (i.e. a $1 \sigma$ uncertainty) for the model parameters for each of the flare components.

\subsection{Discussing the modeling results}

The adiabatic expansion results in a time difference between the peaks in the VLT and APEX light curves of about $1.5 \pm 0.5 \mathrm{~h}$, and compare well with the values obtained in a global, multiwavelength observing campaign by our team in 2007. Back then, two bright NIR flares were traced by CARMA (Combined Array for Research in mm-wave Astronomy; $100 \mathrm{GHz}$ ) in the US, ATCA (Australia Telescope Compact Array; $86 \mathrm{GHz}$ ) in Australia, and the MAMBO bolometer at the IRAM $30 \mathrm{~m}$ in Spain $(230 \mathrm{GHz}$; first results given by Kunneriath et al. 2008;
Eckart et al. 2008b). This light curve complements our parallel 13, 7, and $3 \mathrm{~mm} \mathrm{VLBA}$ run (Lu et al. 2008b). Other recent simultaneous multi-wavelength observations also indicate the presence of adiabatically expanding source components with a delay between the X-ray and sub-mm flares of about $100 \mathrm{~min}$ (Eckart et al. 2006a; Yusef-Zadeh et al. 2008; Marrone et al. 2008). As pointed out by Eckart et al. (2008a,b), a combination of a temporary accretion disk with a short jet can explain most of the properties associated with infrared/X-ray Sgr A* light curves (Eckart et al. 2008a,b).

The adiabatic expansion model: the May 2007 polarimetric NIR measurements (Eckart et al. 2008a) showed a flare event with the highest sub-flare contrast observed until now. In the relativistic disk model these data provide evidence of a spot expansion and its shearing due to differential rotation. An expansion by only $30 \%$ will lower the Synchrotron-Self-Compton (SSC) $\mathrm{X}$-ray flux significantly. In the framework of the spot model this flare event provides additional support for expansion of individual source components.

The expansion speed: the rapid decay of the observed NIR/X-ray flares (e.g. Baganoff et al. 2001; Genzel et al. 2003; Ghez et al. 2004; Eckart et al. 2006a-c), as well as the current results from coordinated observing campaigns including submm monitoring (Eckart et al. 2006b; Yusef-Zadeh et al. 2006b; Marrone et al. 2008, and this work) suggest that non-radiative cooling processes, such as adiabatic expansion, are essential, although the adiabatic cooling model results in very low expansion speeds. From modeling the mm-radio flares Yusef-Zadeh et al. (2008) invoke expansion velocities in the range from $v_{\exp }=$ $0.003-0.1 c$. This compares well with the expansion velocity of the order of $0.0045 \pm 0.0020 \mathrm{c}$ that we obtain in our case with the June 2008 data (see Table 4). These velocities are low compared to the expected relativistic sound speed in orbital velocity in the vicinity of the SMBH. The low expansion velocities suggest that the expanding gas can not escape from $\mathrm{Sgr} \mathrm{A}^{*}$ or must have a large bulk motion (see discussions in Marrone et al. 2008; and Yusef-Zadeh et al. 2008). Therefore the adiabatically expanding source components either have a bulk motion larger than $v_{\exp }$ or the expanding material contributes to a corona or disk, confined to the immediate surroundings of Sgr A*. An expansion of source components through shearing due to differential rotation within the accretion disk may explain the low expansion velocities. The recent theoretical approach of hot spot evolution due to shearing is highlighted in Eckart et al. (2008a) and Zamaninasab et al. (2008; see also Pecháček et al. 2008)

Structure of the light curve: the flux density variations of Sgr A* can be explained in a disk or jet model (see e.g. discussion in Eckart et al. 2006a,b, 2008a), or they could be seen as a consequence of an underlying physical process that can mathematically be described as red-noise (Do et al. 2008; Meyer et al. 2008). In our case we find a light curve structure that consists of maxima separated by about 70 to $110 \mathrm{~min}$ with additional fluctuations of smaller amplitude. Assuming the presence of a disk and by simultaneous fitting of the previously obtained light curve fluctuations and the time-variable polarization angle, we have shown that the data can be successfully modeled with a simple relativistic hot spot/ring model (Meyer et al. 2006a,b, 2007; Eckart et al. 2006a,b, 2008a,b). In this model the broad near-infrared flares ( 100 min duration) of Sgr $\mathrm{A}^{*}$ are due to a 
sound wave that travels around the SMBH once. The sub-flares, superimposed on the broad flare, are thought to be due evolving (i.e. expanding) hot-spots that may be relativistically orbiting the central SMBH. The spot emission would then be due to transiently heated and accelerated electrons which can be modeled as a plasma component (scenarios in which spiral wave structures contribute to the observed variability are also under discussion, e.g. Karas et al. 2007).

In case of a jet (see e.g. Markoff et al. 2007, 2005), the observed flux density variations may more likely be a result of the variations in the accretion process (or jet instabilities) - possibly followed by an adiabatic expansion of the jet components rather than being a result of a modulation from an orbiting spot. In this case one may expect that red-noise variations on these short times scales are a natural extension of the variability found for longer periods.

\section{Summary and discussion}

We have presented new simultaneous measurements of the near-IR sub-mm flare emission of the Sgr A* counterpart associated with the SMBH at the Galactic center. The data were obtained in a global campaign carried out on 3 June 2008, using NACO at VLT and LABOCA at APEX.

The highly variable near-IR emission presents four major events (I-IV), whose peaks are separated by about $\sim 80 \mathrm{~min}$. The peak of the sub-mm light curve is delayed with respect to that of the near-IR data. We argue that this delay is due to the adiabatic expansion of synchrotron source components that become optically thin. The expansion velocity is about $1500 \mathrm{~km} \mathrm{~s}^{-1}(0.5 \%$ of the speed of light). We model the four flare events with 4 source components. In this case the sub-mm light curves of the individual components are blended, with a time delay for the peaks of about $1.5 \pm 0.5 \mathrm{~h}$ with respect to the near-IR data.

The light curve structure is consistent with the previously found variability. It could be interpreted as emission from material in relativistic orbits around the SMBH. However, variable emission from a jet or an explanation as a dominant flux density contribution from a red-noise process cannot be excluded as well.

These data show that the VLT/APEX combination is especially well suited for very long simultaneous light curves between the NIR and the sub-mm domain. Further simultaneous radio/sub-mm data, NIR $K$ - and $L$-band measurements in combination with X-ray observations should lead to a set of light curves that will allow us to prove the proposed model and to discriminate between the individual higher and lower energy flare events. Here Chandra's high angular resolution is ideally suited to separate for weak flares the thermal non-variable bremsstrahlung and the non-thermal variable part of the Sgr A* $\mathrm{X}$-ray flux density.

Acknowledgements. We are grateful to all the ESO PARANAL and Sequitor staff, and especially to the members of the NAOS/CONICA, VLTI, and APEX team. The observations were made possible through a special effort by the APEX/ONSALA staff to have the LABOCA bolometer ready for triggering. Leo Meyer is supported by the DAAD exchange program. Macarena García-Marín is supported by the German federal department for education and research (BMBF) under the project numbers: 500 OS0502 \& 50OS0801. The X-ray work was supported by NASA through Chandra award G05-6093X. M. Zamaninasab, D. Kunneriath, and R.-S. Lu, are members of the International Max Planck Research School (IMPRS) for Astronomy and Astrophysics at the MPIfR and the Universities of Bonn and Cologne. R. Schödel acknowledges support by the Ramón y Cajal programme by the Ministerio de Ciencia e Innovación of the government of Spain. We also thank the referee for his constructive comments.

\section{References}

Baganoff, F. K., Bautz, M. W., Brandt, W. N., et al. 2001, Nature, 413, 45 Blum, R. D., Sellgren, K., \& Depoy, D. L. 1996, ApJ, 470, 864 Diolaiti, E., Bendinelli, O., Bonaccini, D., et al. 2000, A\&AS, 147, 335 Do, T., Ghez, A. M., Morris, M. R., et al. 2008, ApJ, in press [arXiv: 0810.0446]

Eckart, A., \& Genzel, R. 1996, Nature, 383, 415

Eckart, A., \& Genzel, R. 1997 , MNRAS, 284, 576

Eckart, A., Genzel, R., Ott, T., \& Schödel, R. 2002, MNRAS, 331, 917

Eckart, A., Baganoff, F. K., Morris, M., et al. 2004, A\&A, 427, 1

Eckart, A., Baganoff, F. K., Schödel, R., et al. 2006a, A\&A, 450, 535

Eckart, A., Schödel, R., Meyer, L., Trippe, S., et al. 2006b, A\&A, 455, 1

Eckart, A., Schödel, R., Meyer, L., et al. 2006c, ESO Messenger, 125, 2

Eckart, A., Baganoff, F. K., Zamaninasab, M., et al. 2008a, A\&A, 479, 625

Eckart, A., Schödel, R., Baganoff, F. K., et al. 2008b, JPhCS, in press

Eisenhauer, F., Schödel, R., Genzel, R., et al. 2003, ApJ, 597, L121

Eisenhauer, F., Genzel, R., Alexander, T., et al. 2005, ApJ, 628, 246

García-Marín, M. 2008, in prep.

Genzel, R., Schödel, R., Ott, T., et al. 2003, Nature, 425, 934

Ghez, A., Morris, M., Becklin, E. E., Tanner, A., \& Kremenek, T. 2000, Nature, 407,349

Ghez, A. M., Wright, S. A., Matthews, K., et al. 2004, ApJ, 601, 159 Ghez, A. M., Salim, S., Hornstein, S. D., et al. 2005a, ApJ, 620, 744 Ghez, A. M., Hornstein, S. D., Lu, J. R., et al. 2005b, ApJ, 635, 1087 Gierlinski, M., Middleton, M., Ward, M., \& Done, C. 2008, Nature, 455, 369 Gillessen, S., Eisenhauer, F., Quataert, E., et al. 2006, JPhCS, 54, 411 Gould, R. J. 1979, A\&A, 76, 306

Karas, V., Dovciak, M., Eckart, A., Meyer, L., Proc. of the Workshop on the Black Holes and Neutron Stars, ed. S. Hledik, \& Z. Stuchlik, 19-21 September 2007 (Silesian University, Opava) [arXiv: 0709.3836]

Hornstein, S. D., Matthews, K., Ghez, A. M., et al. 2007, ApJ, 667, 900

Hornstein, S. D., Matthews, K., Ghez, A. M., et al. 2006, JPhCS 54, 399 Krabbe, A., Iserlohe, C., Larkin, J. E., et al. 2006, ApJ, 642, L145 Kunneriath, D., et al. 2008, JPhCS, in press

Lu, J. R., Ghez, A. M., Hornstein, S. D., et al. 2008a, ApJ, accepted [arXiv: 0808.3818]

Lu, R.-S., et al. 2008b, JPhCS, in press

Markoff, S., Bower, G. C., \& Falcke, H. 2007, MNRAS, 379, 1519

Markoff, S., Nowak, M. A., \& Wilms, J. 2005, ApJ, 635, 1203

Marrone, D. P., Baganoff, F. K., Morris, M., et al. 2008, ApJ, 682, 373

Marscher, A. P. 1983, ApJ, 264, 296

Mauerhan, J. C., Morris, M., Walter, F., \& Baganoff, F. K. 2005, ApJ, 623, L25

Meyer, L., Eckart, A., Schödel, R., et al. 2006a, A\&A, 460, 15

Meyer, L., Schödel, R., Eckart, A., et al. 2006b, A\&A, 458, L25

Meyer, L., Schödel, R., Eckart, A., et al. 2007, A\&A, 473, 707

Meyer, L., Do, T., Ghez, A., et al. 2008, ApJ, submitted

Pecháček, T., Karas, V., \& Czerny, B. 2008, A\&A, 487, 815

Schödel, R., Ott, T., Genzel, R., et al. 2002, Nature, 419, 694

Reid, M. J. 1993, ARA\&A, 31, 345

Siringo, G., Weiss, A., Kreysa, E., et al. 2007, ESO Messenger, 129, 2

van der Laan, H. 1966, Nature, 211, 1131

Weiss, A., Kovacs, A., Guesten, R., et al. 2008, A\&A, 490, 77

Yusef-Zadeh, F., Wardle, M., Cotton, W. D., Heinke, C. O., \& Roberts, D. A. 2007, ApJ, 668, L47

Yusef-Zadeh, F., Bushouse, H., Dowell, C. D., et al. 2006a, ApJ, 644, 198

Yusef-Zadeh, F., Roberts, D., Wardle, M., Heinke, C. O., \& Bower, G. C. 2006b, ApJ, 650, 189

Yusef-Zadeh, F., Wardle, M., Heinke, C., et al. 2008, ApJ, 682, 361

Zamaninasab, M., Eckart, A., Meyer, L., et al. 2008, Proc. of a conference on Astrophysics at High Angular Resolution (AHAR 08) held 21-25 April 2008 in Bad Honnef, Germany [arXiv : 0810.0138] 